\title{
Photometric parallaxes of Southern high proper motion stars. I.
}

\author{
E. Costa ${ }^{1}$ and R. A. Méndez ${ }^{2}$ \\ 1 Departamento de Astronomía, Universidad de Chile, Casilla 36-D, Santiago, Chile \\ 2 European Southern Observatory, Casilla 19001, Santiago 19, Chile
}

Received 25 October 2002 / Accepted 7 January 2003

\begin{abstract}
Photometric distances derived from VRI photometry are given for 44 high proper motion stars from the Cerro El Roble proper motion surveys of Wroblewski \& Torres and Wroblewski \& Costa. The observations were carried out with the main purpose of estimating the distance of selected members of the above survey that are likely to be nearby. We have discovered 13 stars closer than 25 pc, the classical limit of the Catalogs of Nearby Stars (see e.g. Gliese \& Jahrei $\beta$ 1991) and the NASA/NSF NStars Project. Those stars of the sample whose photometric distances are less than $\sim 25 \mathrm{pc}$, or have proper motions in excess of 0.50 arcsec/year, are either currently being targeted by the Cerro Tololo Parallax Investigation (CTIOPI) or will be targets of a forthcoming expansion of this survey.
\end{abstract}

Key words. astrometry - stars: kinematics - techniques: photometric

\section{Introduction}

The nearest stars, being the brightest examples of their types, provide astronomers with much of our understanding of stellar astronomy. For most types of stars, the fundamental framework of stellar astronomy is built upon direct measurements of luminosities, colors, temperatures, and masses of nearby stars.

The faint members of the solar neighborhood are however significantly underrepresented. Using the Research Consortium on Nearby Stars updated list of stars closer than $10 \mathrm{pc}$ (see Henry 2003), and assuming that the density of stellar systems within $5 \mathrm{pc}$ (44 systems) carries to $10 \mathrm{pc}$, it can be estimated that $125(36 \%)$ systems are missing from the current $10 \mathrm{pc}$ census. The problem is obviously worse to $25 \mathrm{pc}$, a distance at which the incompleteness can be predicted to be as much as $60 \%$.

Unfortunately there is no simple way to deal with this situation; only large trigonometric parallax programs could remedy the problem. Furthermore, for such surveys to be efficient, their input target lists must be as refined as possible, so candidate nearby stars must be carefully selected on the basis of some "closeness" indicator; such as having a high proper motion and/or a photometric estimate of their distance.

With the above immediate purpose in mind, and within the scope of the Cerro Tololo Parallax Investigation (CTIOPI, Henry et al. 1999), a southern hemisphere trigonometric parallax survey whose goal is to increase the population of stars known within $25 \mathrm{pc}$ of the Sun by $20 \%$, along with

Send offprint requests to: E. Costa,

e-mail: costa@das.uchile.cl a forthcoming expansion of the CTIOPI effort, we started a program to determine photometric distances of high proper motion stars from the Cerro El Roble (EACR) proper motion surveys of Wroblewski \& Torres (hereafter W\&T, see e.g. A\&AS 122, $447,1997)$ and Wroblewski \& Costa (hereafter W\&C, see e.g. A\&A 367, 725, 2001).

In the course of the EACR surveys, 2495 new stars with proper motions larger than 0.15 arcsec/year were discovered and 1262 Luyten Catalogue (LTT, Luyten 1957) were rediscovered, so, for our program to be realistic, it was necessary to pre-select the candidates for the photometry. This was done essentially on the basis of their proper motion, the faster being more important. Because of their possible astrophysical importance, the highest ranked objects were those that are both fast moving and faint. After excluding objects with known parallaxes (mainly bright stars), and those being targeted by the photometric survey of Ianna et al. (see e.g. Patterson et al. 1998), a priority ranking including some 200 stars was created. It should be noted that roughly $10 \%$ of the stars in our working list are currently being observed by CTIOPI, and a similar additional percentage will be targeted by the forthcoming extension of this survey.

In this first paper we give photometric distances, based on VRI photometric observations made with the $0.9 \mathrm{~m}$ telescope Cerro Tololo Interamerican Observatory (CTIO), for part of the brighter sample of WT/LTT stars in our list. Coordinates and finding charts for the 44 objects observed can be found as indicated in Table 1 - in the series of papers of the EACR survey (it should be noted that the LTT catalogue does not include finders). In a second contribution being prepared, we will 
present results for the fainter sample, based mainly on observations carried out with the CTIO $1.5 \mathrm{~m}$ telescope.

\section{Observations}

The observations were made with a Tektronic $2048 \times 2046$ CCD detector with $24 \mu$ pixels attached to the Cassegarin focus of the CTIO $0.9 \mathrm{~m}$ telescope. This combination gives a field size of $13.5^{\prime} \times 13.5^{\prime}$ and a scale of $0.396^{\prime \prime} /$ pixel. The inverse gain was $\sim 1.5 \mathrm{e}-/ \mathrm{ADU}$, and the corresponding read noise $\sim 3.6 \mathrm{e}-$.

Our VRI instrumental photometric system was defined by the use of the following filters: Vtek2: 5475/1000, Rtek2: 6425/1500 and I-KC\#31: 8075/1500 (where the first figure is the measured central wavelength of the filter and the second its $F W H M$, in A). These filters constitute the default option on the $0.9 \mathrm{~m}$ telescope for broad-band photometry on the standard VRI Johnson-Kron-Cousins system. Additional information about them, including their transmission curves, can be found in http://www.ctio.noao.edu/instruments/filters/ index.html

Typically $6 U B V R I$ standard star areas from the catalogs of Landolt (1992) and Graham (1982) were observed multiple times each night to determine the transformation of our instrumental magnitudes to the standard VRI system. Although most of these areas include stars of a wide variety of colors, given the presumably very red colors of most of our targets, very red standards were observed additionally. A few of the standard areas were followed each night up to about 2.2 airmasses to optimally determine atmospheric extinction.

All program stars were observed during transit, following the sequence VRIIRV. Although time consuming, this latter practice has proved very useful to check for consistency.

\section{Reductions}

All CCD frames were first calibrated using standard IRAF (version 2.11.3, NOAO, University of Arizona) tasks. For this purpose Zero exposures and Twilight Sky Flats were taken every night.

Aperture photometry was then performed on each object of interest using the IRAF APPHOT package. The optimum aperture size for each night, ensuring a negligible loss of light from the PSF wings and minimizing light contamination from close objects, was determined by means of the IRAF MKAPFILE task. The best aperture radius turned out to be 4-5 times the average $F W H M$ of the frames.

To put our observations into the standard system, we used the following transformation equations:

$$
\begin{aligned}
v & =V+v_{1}+v_{2} \cdot X_{v}+v_{3} \cdot(V-R)+v_{4} \cdot(V-R) \cdot X_{v} \\
r & =R+r_{1}+r_{2} \cdot X_{r}+r_{3} \cdot(V-R)+r_{4} \cdot(V-R) \cdot X_{r} \\
i & =I+i_{1}+i_{2} \cdot X_{i}+i_{3} \cdot(V-I)+i_{4} \cdot(V-I) \cdot X_{i}
\end{aligned}
$$

where $(v, r, i)$ and $(V, R, I)$ are the instrumental and standard magnitudes respectively, $\left(X_{v}, X_{r}, X_{i}\right)$ are the airmasses and $v_{1}$, $v_{2}, v_{3}, v_{4}$, etc. are constants. It should be noted that in the absence of blue-passband observations it was not possible to use (as is the common practice) the $(B-V)$ color for the standardization of the $\mathrm{v}$ magnitude. As shown previously by Bucciarelli et al. (2001), the $(B-V)$ and $(V-R)$ colors of the Landolt stars are linearly related and comparable in range, so the use of the $(V-R)$ color in the $(v, V)$ equation is equally satisfactory.

Equations (1) were applied to the Landolt/Graham standard star magnitudes, and solved using the IRAF FITPARAMS task, which performs a least-square fit to the system. This task can be run interactively, which permits the rejection of problematic observations to control the quality of the fit. Without major massaging, the rms of all fits turned out to be around $0.02 \mathrm{mag}$. With the exception of the second-order color terms coefficients $\left(v_{4}, r_{4}, i_{4}\right)$ the formal errors of the calculated coefficients were significantly smaller than their derived values.

Finally, the above set of transformation equations - with their corresponding calculated coefficients - was applied to our program stars for their calibration. This was done by means of the IRAF INVERTFIT task, which produces a set of calibrated magnitudes and colors and their errors: in the present case specifically: $V$, error $(V),(V-R)$, error $(V-R)$ and $(V-I)$, error $(V-I)$. As pointed out by Bucciarelli et al. (2001), the final photometric error computed by INVERTFIT does not rigorously treat error propagation, therefore producing a lower limit of the photometric errors.

\section{The photometry}

The results of our VRI photometry are presented in Table 1. The first column gives the name of the star in the EACR survey (the "WT" number) or its name in the LTT Catalogue; the second column the number of times the star was observed; Cols. 3, 6 and 9 their average $V$ magnitude and $(V-R)$ and $(V-I)$ colors, respectively; Cols. 4, 7 and 10 the square root of the sum of the squares of the IRAF-computed errors of each observation for $V,(V-R)$ and $(V-I)$, respectively; Cols. 5, 8 and 11 the standard deviation of the average values of $V,(V-R)$ and $(V-I)$, respectively. These errors have to be interpreted with caution; it must be kept in mind that they have been derived from a small number of independent measures. They have been included here for comparison with the lower limit error given by IRAF, and because they are the starting point to determine the propagation of errors described in Sect. 6. It should also be noted that high photometric errors in $(V-R)$ and $(V-I)$ usually just reflect a poor determination of the $V$ magnitude; this is because - these stars being in general quite brighter in the $R$ and $I$ bandpasses - their $R, I$ magnitudes are precisely determined. Finally, Col. 12 gives the reference to the W\&T or $\mathrm{W} \& \mathrm{C}$ paper where coordinates, proper motion, position angle, and a finding chart can be found for the star. An asterisk in the Remarks column indicates that additional comments are made in Sect. 5 (Notes on individual objects).

\section{Notes on individual objects}

WT233: two objects are now seen near the position indicated for this star by the W\&T finding chart (see W\&T, A\&AS 91, 129, 1991). The W\&T finder was made from a Maksutov Astrograph plate (scale: 99.4" $/ \mathrm{mm}$ ) taken circa 1990, and 
Table 1. VRI Photometry of Southern high proper motion stars from the Cerro El Roble proper motion survey.

\begin{tabular}{|c|c|c|c|c|c|c|c|c|c|c|c|c|}
\hline STAR & $N_{\text {obs }}$ & V & $E r r_{V}$ & $\sigma_{V}$ & $V-R$ & $\operatorname{Err}_{(V-R)}$ & $\sigma_{(V-R)}$ & $V-I$ & $\operatorname{Err}_{(V-I)}$ & $\sigma_{(V-I)}$ & Ref. & Remarks \\
\hline WT98 & 5 & 14.023 & 0.002 & 0.027 & 1.203 & 0.002 & 0.028 & 2.627 & 0.002 & 0.018 & 1 & \\
\hline WT110 & 4 & 16.932 & 0.017 & 0.023 & 1.193 & 0.019 & 0.021 & 2.700 & 0.017 & 0.020 & 1 & \\
\hline WT121 & 4 & 15.587 & 0.005 & 0.039 & 1.274 & 0.006 & 0.036 & 2.884 & 0.006 & 0.048 & 1 & \\
\hline WT133 & 6 & 16.021 & 0.008 & 0.018 & 1.350 & 0.008 & 0.020 & 3.064 & 0.008 & 0.018 & 1 & \\
\hline WT135 & 7 & 14.161 & 0.002 & 0.024 & 1.099 & 0.002 & 0.030 & 2.338 & 0.002 & 0.023 & 1 & \\
\hline WT143 & 4 & 16.325 & 0.011 & 0.053 & 0.805 & 0.013 & 0.043 & 1.518 & 0.013 & 0.057 & 1 & \\
\hline WT178 & 4 & 14.919 & 0.003 & 0.011 & 1.402 & 0.004 & 0.007 & 3.121 & 0.003 & 0.004 & 3 & \\
\hline WT233b & 6 & 16.235 & 0.008 & 0.042 & 0.920 & 0.009 & 0.029 & 1.821 & 0.009 & 0.023 & 3 & $*$ \\
\hline WT233f & 6 & 16.901 & 0.014 & 0.050 & 0.469 & 0.018 & 0.063 & 0.993 & 0.021 & 0.104 & 3 & $*$ \\
\hline WT1289 & 5 & 16.181 & 0.011 & 0.049 & 1.265 & 0.012 & 0.037 & 2.881 & 0.011 & 0.039 & 5 & $*$ \\
\hline WT1353 & 4 & 13.916 & 0.002 & 0.028 & 1.083 & 0.003 & 0.009 & 2.347 & 0.003 & 0.009 & 5 & \\
\hline WT1356 & 6 & 16.080 & 0.009 & 0.041 & 1.401 & 0.009 & 0.043 & 3.126 & 0.009 & 0.035 & 5 & \\
\hline WT1463 & 6 & 16.159 & 0.011 & 0.043 & 1.261 & 0.012 & 0.042 & 2.827 & 0.011 & 0.026 & 5 & \\
\hline WT1481 & 6 & 13.622 & 0.002 & 0.017 & 0.703 & 0.002 & 0.019 & 1.328 & 0.002 & 0.011 & 5 & \\
\hline WT1515 & 7 & 14.903 & 0.004 & 0.041 & 1.283 & 0.004 & 0.053 & 2.842 & 0.004 & 0.040 & 5 & \\
\hline WT1530 & 6 & 16.882 & 0.011 & 0.042 & 1.417 & 0.012 & 0.060 & 3.110 & 0.011 & 0.036 & 5 & \\
\hline WT1542 & 6 & 14.116 & 0.002 & 0.035 & 1.230 & 0.002 & 0.045 & 2.698 & 0.002 & 0.037 & 5 & \\
\hline WT1759 & 4 & 15.446 & 0.007 & 0.019 & 0.275 & 0.010 & 0.028 & 0.544 & 0.011 & 0.016 & 6 & \\
\hline WT1760b & 4 & 16.164 & 0.008 & 0.029 & 1.390 & 0.010 & 0.036 & 3.070 & 0.009 & 0.025 & 6 & $*$ \\
\hline WT2390 & 7 & 15.215 & 0.004 & 0.027 & 1.168 & 0.005 & 0.028 & 2.569 & 0.004 & 0.029 & 8 & \\
\hline WT2431 & 6 & 13.708 & 0.002 & 0.024 & 0.966 & 0.002 & 0.024 & 1.897 & 0.002 & 0.017 & 8 & \\
\hline WT2451 & 8 & 15.062 & 0.003 & 0.027 & 1.214 & 0.004 & 0.044 & 2.727 & 0.003 & 0.037 & 8 & \\
\hline WT2461 & 6 & 15.264 & 0.004 & 0.036 & 1.183 & 0.005 & 0.022 & 2.669 & 0.004 & 0.033 & 8 & \\
\hline WT2462 & 4 & 15.089 & 0.005 & 0.016 & 1.318 & 0.006 & 0.020 & 2.957 & 0.005 & 0.015 & 8 & \\
\hline WT2463 & 4 & 15.711 & 0.006 & 0.045 & 1.046 & 0.007 & 0.028 & 2.225 & 0.006 & 0.040 & 8 & \\
\hline WT2475 & 6 & 15.899 & 0.006 & 0.017 & 1.386 & 0.006 & 0.014 & 3.082 & 0.006 & 0.014 & 6 & \\
\hline LTT1323 & 4 & 13.138 & 0.001 & 0.029 & 1.141 & 0.001 & 0.015 & 2.550 & 0.001 & 0.016 & 2 & \\
\hline LTT1588 & 7 & 11.495 & 0.001 & 0.011 & 0.407 & 0.001 & 0.007 & 0.784 & 0.001 & 0.006 & 8 & $*$ \\
\hline LTT1697 & 6 & 12.801 & 0.001 & 0.022 & 0.932 & 0.001 & 0.006 & 1.814 & 0.001 & 0.015 & 8 & \\
\hline LTT1848 & 4 & 13.012 & 0.001 & 0.017 & 1.029 & 0.001 & 0.023 & 2.128 & 0.001 & 0.024 & 2 & \\
\hline LTT2202 & 6 & 14.544 & 0.003 & 0.026 & 0.941 & 0.003 & 0.039 & 1.854 & 0.003 & 0.029 & 8 & \\
\hline LTT2631 & 5 & 12.936 & 0.001 & 0.010 & 1.183 & 0.002 & 0.011 & 2.656 & 0.002 & 0.015 & 7 & \\
\hline LTT2856 & 6 & 12.587 & 0.001 & 0.028 & 1.068 & 0.001 & 0.011 & 2.290 & 0.001 & 0.011 & 4 & \\
\hline LTT3461 & 6 & 11.235 & 0.001 & 0.013 & 0.864 & 0.001 & 0.008 & 1.677 & 0.001 & 0.007 & 7 & \\
\hline LTT3496 & 6 & 15.094 & 0.003 & 0.029 & 0.138 & 0.004 & 0.019 & 0.317 & 0.006 & 0.029 & 4 & \\
\hline LTT3553 & 6 & 12.590 & 0.001 & 0.034 & 1.081 & 0.001 & 0.014 & 2.383 & 0.001 & 0.018 & 7 & \\
\hline LTT3616 & 6 & 11.882 & 0.001 & 0.020 & 1.064 & 0.001 & 0.014 & 2.349 & 0.001 & 0.011 & 7 & \\
\hline LTT3632 & 6 & 12.958 & 0.001 & 0.014 & 1.101 & 0.001 & 0.017 & 2.436 & 0.001 & 0.030 & 4 & \\
\hline LTT3725 & 4 & 15.650 & 0.006 & 0.007 & 1.171 & 0.007 & 0.010 & 2.653 & 0.007 & 0.057 & 8 & \\
\hline LTT3742 & 4 & 13.021 & 0.001 & 0.013 & 1.163 & 0.001 & 0.011 & 2.555 & 0.002 & 0.013 & 8 & \\
\hline LTT3790 & 4 & 11.673 & 0.001 & 0.006 & 1.044 & 0.001 & 0.009 & 2.194 & 0.001 & 0.015 & 4 & \\
\hline LTT3791 & 4 & 13.202 & 0.002 & 0.005 & 1.240 & 0.003 & 0.007 & 2.756 & 0.002 & 0.010 & 4 & \\
\hline LTT3802 & 5 & 11.274 & 0.001 & 0.001 & 0.976 & 0.001 & 0.012 & 1.940 & 0.001 & 0.021 & 7 & \\
\hline LTT3861 & 6 & 11.211 & 0.001 & 0.008 & 0.868 & 0.001 & 0.010 & 1.640 & 0.001 & 0.014 & 8 & \\
\hline
\end{tabular}

References to coordinates, proper motions, and finding charts.

[1] Wroblewski, H., \& Torres, C. 1989, A\&AS, 78, 231

[2] Wroblewski, H., \& Torres, C. 1990, A\&AS, 83, 317

[3] Wroblewski, H., \& Torres, C. 1991, A\&AS, 91, 129

[4] Wroblewski, H., \& Torres, C. 1992, A\&AS, 92, 449

[5] Wroblewski, H., \& Torres, C. 1996, A\&AS, 115, 481

[6] Wroblewski, H., \& Torres, C. 1997, A\&AS, 122, 447

[7] Wroblewski, H., \& Torres, C. 1998, A\&AS, 128, 457

[8] Wroblewski, H., \& Costa, E. 2001, A\&A, 367, 725.

shows only one object; because at that epoch one star was eclipsing the other. On account of the proper motion that occurred in the roughly 10 year epoch difference between that plate and our photometry (plus the better scale of the photome- try frames), two objects are now clearly distinguished. We identify the brightest and reddest of them, "WT233b" in Tables 1 and 2, as the correct W\&T star. The fainter object, "WT233f" in those tables, lies $\sim 9$ " to the SE. This object is bluer and seems 


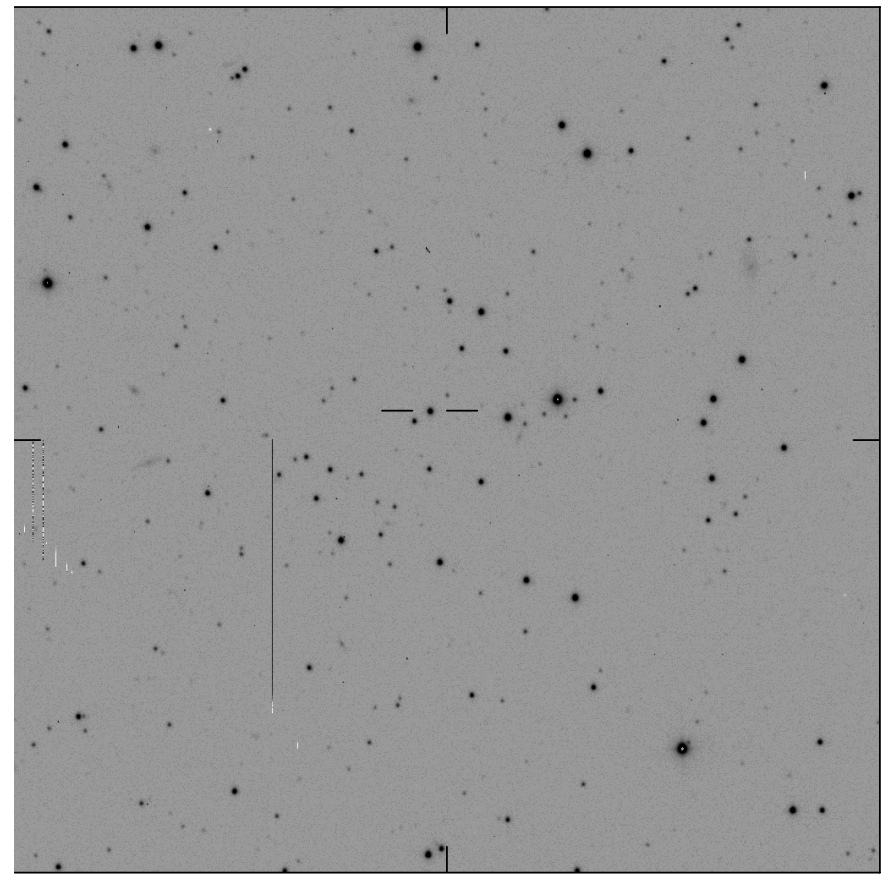

Fig. 1. Finding chart for WT233. Chart is 4.1 arcmin on a side; North is at the top, East to the left. The object marked with ticks, which we identify as the W\&T star, is WT233b in Tables 1 and 2. The fainter object immediately to the SE is WT233f. See Sect. 5 for details.

to be variable. It is interesting to note that the variability is most notable in the $I$ and $R$ bandpasses. In Fig. 1 we give a finding chart produced from a photometry frame.

WT1289: there is a very faint red object $\sim 7^{\prime \prime}$ to the SE of this star, which is not visible in the W\&T finder. Photometry was not possible due to its faintness.

WT1760: two objects are now seen near the position indicated for this star by the W\&T finding chart (see W\&T, A\&AS 122, $447,1997)$. The W\&T finder was made from an image extracted from the Digitized Sky Survey (produced by the Space Telescope Science Institute), which was in turn obtained from a UK Schmidt Telescope plate (scale: $67.2^{\prime \prime} / \mathrm{mm}$ ) taken circa 1980, and shows one object because at that epoch one star was eclipsing the other. On account of the proper motion that occurred in the roughly 20-year epoch difference between that plate and our photometry (plus the better scale of the photometry frames), two objects are now clearly distinguished. We identify the brighter and much redder object, "WT1760b" in Tables 1 and 2, as the correct W\&T star. The bluer and fainter object (not included in those tables), lies $\sim 11^{\prime \prime}$ to the NW. For this latter, which seems to be variable, we obtained moderate precision photometry: $V=16.94$, error $(V)=0.04$; $(V-R)=0.42$, error $(V-R)=0.06 ;(V-I)=0.81$, error $(V-I)=0.09$ (IRAF-computed errors). In Fig. 2 we give a finding chart produced from a photometry frame.

LTT1588: there is a moderately faint red object, clearly visible in the W\&C finder, $\sim 10^{\prime \prime}$ to the NE of this star. We could only secure low-precision photometry of it: $V=16.63$, error $(V)=0.11 ;(V-R)=0.97$, error $(V-R)=0.12 ;(V-I)=1.96$, error $(V-I)=0.12$ (IRAF-computed errors).

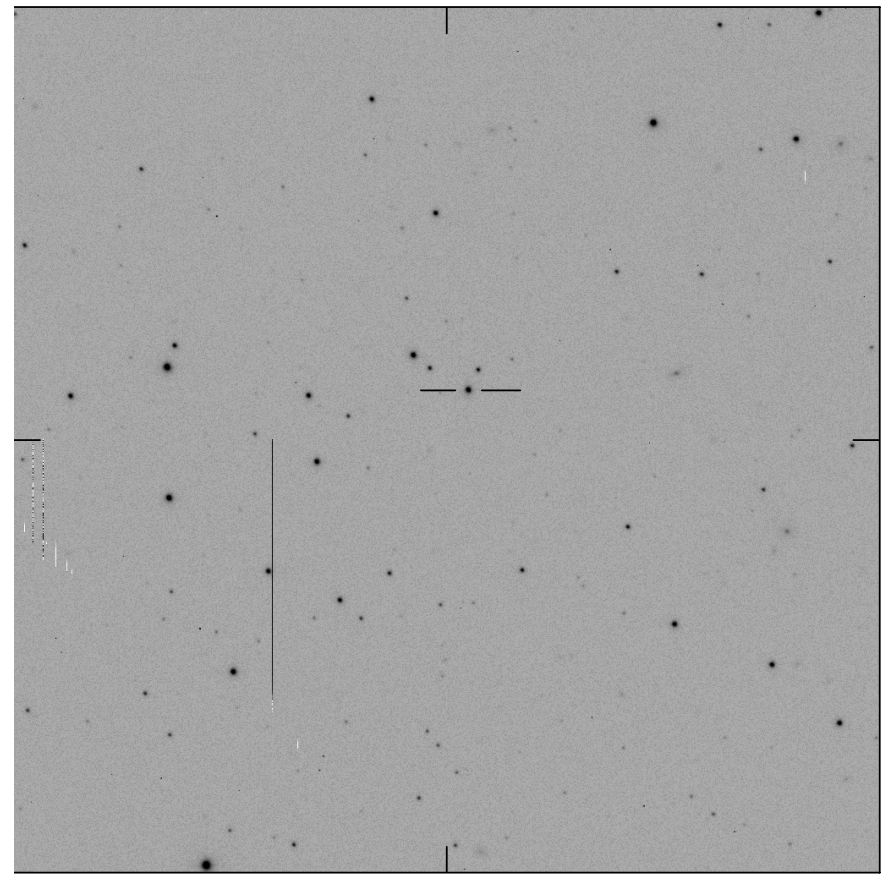

Fig. 2. Finding chart for WT1760. Chart is 4.1 arcmin on a side; North is at the top, East to the left. The object marked with ticks, which we identify with the W\&T star, is WT1760b in Tables 1 and 2. The fainter object immediately to the NW is that alluded to in Sect. 5 .

\section{Photometric distances}

In this section we discuss the methods employed to obtain photometric parallaxes, using the VRI photometry described in the previous sections.

Photometric distances have been determined in two semiindependent ways: using a relationship between $M_{V}$ and $(V-R)$ on one hand, and between $M_{V}$ and $(V-I)$ on the other. These relationships have been derived by T. Henry (private communication), using the latest data available in the Research Consortium on Nearby Stars (RECONS) database, which contains trigonometric parallaxes and good photometric data for about 140 stars closer than $10 \mathrm{pc}$ (see http://www. chara.gsu. edu/ thenry/RECONS). The above relationships are valid in the spectral type range from about G5 to M8, which corresponds to a range of $0.37-2.20$ in $(V-R)$, a range of 0.55-4.70 in $(V-I)$, and an absolute magnitude range of $4.0 \leq M_{V} \leq 18.0$. As shown by Table 1 , some stars in our sample have colors slightly bluer than those allowed by the relationships derived by Henry; in these few cases we used fits derived from the tabulations provided by Schmidt-Kaler (1986, Table 13) and Schneider (1996, Table 9) to obtain $M_{V}$.

Given the faintness and large proper motion of the stars of our sample, it is very unlikely that there are giants or sub-giants among them; but, since all of the above relationships are valid only for main-sequence stars, before deriving the photometric distances of our targets we verified that they indeed appear to satisfy the above relationships. This was accomplished by plotting our targets on a color-color diagram, and superimposing fiducial lines for main sequence stars, the result of which is presented in Fig. 2. As can be seen from this figure, all our 


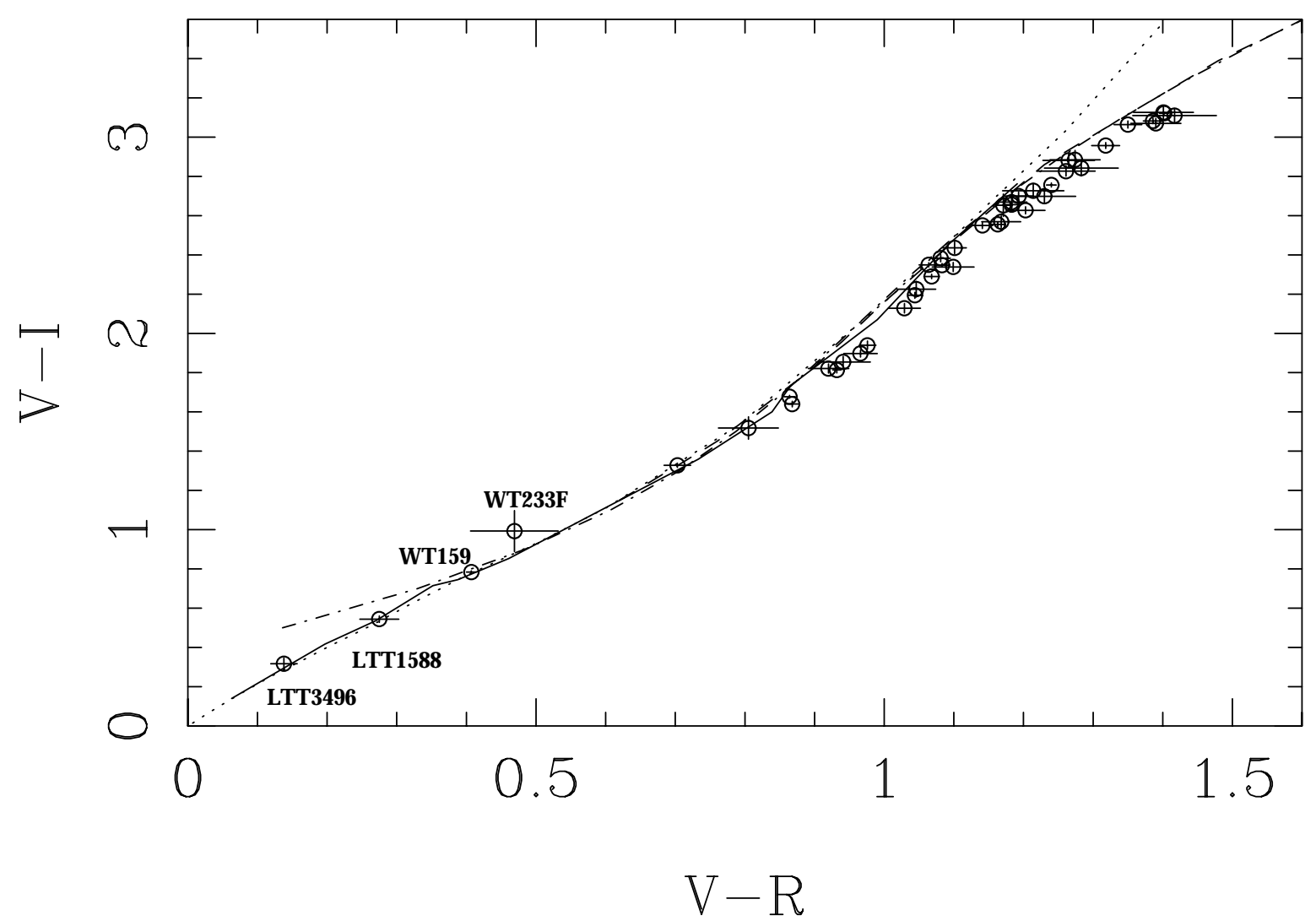

Fig. 3. Color-color diagram for our stars compared to various fiducial lines. Note the unequal size of both axis, which makes it appear as if the errors in $(V-R)$ are larger than those in $(V-I)$, whereas in fact they are comparable. The solid line comes from Table 9 in Schneider (1996), which gives colors computed from Vilnius spectra. The dashed line is from Bessell (1995), and it is based on a combination of model and empirical model atmospheres for mid-K to late M-type stars. The dot-dashed line is from the Palomar/MSU spectroscopic survey of nearby stars by Hawley's et al. (1996), while the dotted line is from an empirical calibration presented by Drilling \& Landolt (2000, Table 15.3.3). As it can be seen, the agreement between all these fiducial lines and our data is quite good, and within our observational errors. The four blueish stars marked in the plot are discussed further in the text.

stars follow the expected main sequence locus, and thus, it is justified to apply the relationships mentioned above to derive their absolute magnitudes.

Since we have data with various degrees of accuracy in its photometry (see Table 1), we have used a Monte-Carlo approach to derive the photometric distances. Each star has a mean color and an associated error for this color. These two values are used in a simulation to derive many plausible photometric distances. After an appropriate number of simulations has been done (defined as the number of simulations for which both the mean distance and the distance dispersion does not change significantly as we run more simulations, see below), a mean value and a scatter for the distance is computed. The final scatter in the derived distances is thus related to the accuracy in the input photometric data exclusively.

In this method we assume that the absolute magnitude vs. color relationship has a scatter smaller than that introduced by the accuracy of our photometry. While this might be true for stars of a given (fixed) metallicity, the natural spread in metallicity for Disk stars adds an extra scatter, which can account for up to 0.2-0.3 mag in $M_{I}$ for a given $(V-I)$ (Leggett 1992; Kirkpatrick et al. 1994). This "cosmic" scatter, can therefore add a extra $\sim 14 \%$ error to the derived photometric distances.
This is further confirmed by Henry (2003), who made a comparison of photometric and trigonometric distances for 97 stars within $10 \mathrm{pc}$, which indicates that the distance estimation technique utilized here results in distances accurate to about $15 \%$. However, since we have no metallicity indicator, we do not have a way to account for this effect.

The Monte-Carlo simulations mentioned above follow the same methods outlined in Méndez \& Ruiz (2000), although used in a quite different context. Details of the simulations can be found in that publication. Briefly, these simulations are important because of the obvious non-linear relationship between photometric errors and (photometric) distance errors. For each pair $\left(C O L, \sigma_{C O L}\right)$, we generate many random colors following a Gaussian distribution of width $\sigma_{C O L}$ centered around COL. For each of these, a photometric distance is calculated. After many distances have been generated in this way, a mean distance and an rms distance is calculated, and this is repeated for every star. More specifically, and following Méndez \& Ruiz (2000), the value adopted for the color is given by:

$C O L_{i, j}=C O L_{j}+\sigma_{C O L_{j}} \times G_{i}$

where $C O L_{j}$ is the (mean) observed color for star " $j$ " in the sample, with measurement error $\sigma_{C O L_{j}} ; G_{i}$ the Gaussian deviate (of zero mean and unity variance) for simulation " $i$ ", and 
$C O L_{i, j}$ is the $i$ th simulation value for the color of star $j$. The no-errors situation is, of course, reproduced when all the $G_{i}$ are set to zero. The numbers of simulations is chosen such that the variation in the derived mean distance and dispersion is quite small (e.g., less than 1\%), and it is typically less than 100 simulations per star. Table 2 shows our results for the photometric distances and the estimated dispersion as derived from each color; the weighted mean is given in the last two columns.

Inspection of Table 2 shows that, in general, there is a very good agreement between the distances derived from each color separately; in most cases the differences are within $3 \sigma$ of the estimated distance dispersion. We must note however that the two methods for estimating the distances are not completely independent. The $(V-R)$ and $(V-I)$ colors rely on identical $V$ magnitudes, so these colors are semi-independent. While one could argue that the $M_{V}$ values (that are responsible for most of the scatter through its intrinsic metallicity scatter) depend on the same parallax measurement for both relations, it is also evident that one has fitted $M_{V}=V+5 \times \log (10 / D)$ to a set of different values in the ordinate (the colors), such that the fits are actually independent. The differences between distances derived using $(V-R)$ and $(V-I)$ as given in Table 2 allow us to determine a global variance on the mean distances associated with the use of two colors. Excluding the four problematic stars (WT233f, WT1759, LTT1588, and LTT3496; see next paragraph) we obtain an overall standard deviation of $6.7 \mathrm{pc}$, which one could consider as the "intrinsic" distance dispersion of the method (excluding the cosmic variance discussed before). This is fully supported by a comparison to a couple of stars in our sample for which recent parallaxes have become available (see below).

As can be seen from Fig. 1, the three objects with distances larger than $1 \mathrm{kpc}$ (WT233f, WT1759, and LTT3496) have bluish colors indicative of an early spectral type. LTT3496 is indeed a white dwarf (see e.g. Sion et al. 1988), and Henry et al. (2002) recently identified WT1759 as such - and published colors in perfect agreement with ours. This could therefore also be the case of WT233f (please note that this star has large photometric errors and seems to be variable - see Table 1 and Sect. 5), and of another bluish star in our sample, LTT1588 (whose much closer distance is consistent with its brighter apparent magnitude). Of course, another possibility is that they are subdwarfs. In the absence of further colors, a spectroscopic follow-up is necessary to settle this matter. In either case, it must be stressed that for such objects distances and luminosities deduced from VRI photometric observations are not reliable.

Currently, CTIOPI has measured preliminary distances for 2 of the objects included in Table 2, WT133 and LTT2631, for which distances of 36.2 and 19.2 pc were obtained, respectively. Given the limitations of the photometric parallax method, which, as discussed in Sect. 6, go beyond the computed statistical errors, the photometric distances we have determined for WT133 and LTT2631 (31 \pm 1 and $15 \pm 1$ pc, respectively) are in good agreement with the results obtained by CTIOPI. Furthermore, 4 stars of our sample, namely WT133, WT233b, WT1759 and WT1769, have spectroscopic distance estimates by Henry et al. (2002). With the exception of the spectroscopically-confirmed white dwarf WT1759 (whose
Table 2. Photometric distances and its dispersion (all in pc) as derived from data in Table 1.

\begin{tabular}{|c|c|c|c|c|c|c|}
\hline STAR & $D(V-R)$ & $\sigma_{D(V-R)}$ & $D(V-I)$ & $\sigma_{D(V-I)}$ & $D$ & $\sigma_{D}{ }^{*}$ \\
\hline WT98 & 22 & 3 & 27 & 1 & 26 & 1 \\
\hline WT110 & 87 & 9 & 90 & 3 & 89 & 3 \\
\hline WT121 & 32 & 6 & 35 & 3 & 34 & 3 \\
\hline WT133 & 28 & 2 & 31 & 1 & 31 & 1 \\
\hline WT135 & 38 & 6 & 47 & 2 & 46 & 2 \\
\hline WT143 & 388 & 70 & 441 & 37 & 429 & 33 \\
\hline WT178 & 14 & 1 & 17 & 1 & 17 & 1 \\
\hline WT233b & 227 & 30 & 275 & 11 & 269 & 10 \\
\hline WT233f & 2140 & 662 & 1340 & 287 & 1467 & 263 \\
\hline WT1289 & 44 & 8 & 46 & 3 & 46 & 3 \\
\hline WT1353 & 37 & 2 & 41 & 1 & 40 & 1 \\
\hline WT1356 & 24 & 4 & 29 & 2 & 28 & 2 \\
\hline WT1463 & 45 & 9 & 50 & 3 & 50 & 2 \\
\hline WT1481 & 164 & 12 & 165 & 3 & 165 & 3 \\
\hline WT1515 & 23 & 6 & 27 & 2 & 27 & 2 \\
\hline WT1530 & 33 & 8 & 43 & 3 & 41 & 3 \\
\hline WT1542 & 20 & 4 & 25 & 2 & 24 & 2 \\
\hline WT1759 & 2459 & 342 & 2414 & 271 & 2432 & 212 \\
\hline WT1760b & 26 & 4 & 33 & 2 & 32 & 1 \\
\hline WT2390 & 45 & 6 & 51 & 3 & 50 & 3 \\
\hline WT2431 & 58 & 6 & 77 & 2 & 75 & 2 \\
\hline WT2451 & 34 & 7 & 36 & 2 & 36 & 2 \\
\hline WT2461 & 42 & 5 & 44 & 3 & 44 & 2 \\
\hline WT2462 & 21 & 2 & 24 & 1 & 24 & 1 \\
\hline WT2463 & 100 & 13 & 116 & 8 & 117 & 7 \\
\hline WT2475 & 23 & 1 & 28 & 1 & 27 & 1 \\
\hline LTT1323 & 19 & 1 & 20 & 1 & 20 & 1 \\
\hline LTT1588 & 241 & 11 & 171 & 3 & 175 & 3 \\
\hline LTT1697 & 44 & 1 & 57 & 1 & 50 & 1 \\
\hline LTT1848 & 31 & 3 & 39 & 2 & 38 & 1 \\
\hline LTT2202 & 96 & 17 & 120 & 5 & 118 & 5 \\
\hline LTT2631 & 14 & 1 & 15 & 1 & 15 & 1 \\
\hline LTT2856 & 21 & 1 & 25 & 1 & 24 & 1 \\
\hline LTT3461 & 29 & 1 & 34 & 1 & 33 & 1 \\
\hline LTT3496 & 3582 & 202 & 3416 & 166 & 3483 & 128 \\
\hline LTT3553 & 20 & 1 & 21 & 1 & 21 & 1 \\
\hline LTT3616 & 16 & 1 & 16 & 1 & 16 & 1 \\
\hline LTT3632 & 22 & 2 & 23 & 1 & 23 & 1 \\
\hline LTT3725 & 53 & 3 & 54 & 6 & 53 & 2 \\
\hline LTT3742 & 17 & 1 & 19 & 1 & 19 & 1 \\
\hline LTT3790 & 16 & 1 & 19 & 1 & 18 & 1 \\
\hline LTT3791 & 12 & 1 & 15 & 1 & 14 & 1 \\
\hline LTT3802 & 18 & 1 & 24 & 1 & 21 & 1 \\
\hline LTT3861 & 28 & 1 & 35 & 1 & 33 & 1 \\
\hline
\end{tabular}

* Note that this error does not include the possible metallicity effects discussed in the text, which could increase the error of the derived distance up to $14 \%$. 
distance is estimated by Henry et al. by comparison of its spectra with the white dwarf standard GJ 440), the results agree well within the declared uncertainties.

The most interesting result in view of our ultimate objective is that we have discovered 13 stars closer than $25 \mathrm{pc}$, the classical limit of the Catalogs of Nearby Stars (see e.g. Gliese $\&$ Jahrei $\beta$ 1991) and horizon of the NASA/NSF NStars Project. As immediate follow-up observations, we plan to obtain spectra of these promising objects to further constrain their distances via spectral types. The proposed additional observations will also help to fully characterize them.

\section{Conclusions}

As intended, our observations have served the purpose of additionally filtering our list of suspected (on the basis of their high proper motion) nearby stars. From an efficiency viewpoint, this filtering process is mandatory to determine the observational priority that objects of our list could have in any major trigonometric parallax program -such as those mentioned in the introduction- aimed at discovering the "missing" members of the solar neighborhood.

The VRI photometry is furthermore important by itself. In general terms, the photometric results should help to understand the W\&T and W\&C samples in terms of a mixture of stellar populations; and, in the case of those objects that are targets of CTIOPI (or may become targets of future trigonometric parallax programs), their $V R I$ colors are necessary to determine the refraction corrections to be applied to each of them.

Acknowledgements. This work was partially financed by the Fondo Nacional de Investigación Científica y Tecnológica (proyecto No. 1010137 Fondecyt). Edgardo Costa also acknowledges support from the Chilean Centro de Astrofísica FONDAP No. 15010003. Information from additional members of the RECONS group (http://www. chara.gsu.edu/RECONS) has been helpful in preparing this manuscript.

\section{References}

Bessell, M. S. 1995, in The bottom of the Main sequence and beyond, ed. C. G. Tinney (Berlin: Springer-Verlag), 123

Bucciarelli, B., Garcia Yus, J., Casalegno, R., et al. 2001, A\&A, 368, 335

Drilling, J. S., \& Landolt, A. U. 2000, in Allen's Astrophysical Quantities, Fourth Edition, ed. A. N. Cox (New York: SpringerVerlag), 392

Gliese, W., \& Jahrei $\beta$, H., On The Astronomical Data Center CD-ROM: Selected Astronomical Catalogs, vol. I, ed. L. E. Brotzmann, \& S. E. Gesser, NASA/Astronomical Data Center, Goddard Space Flight Center, Greenbelt, MD

Graham, J. A. 1982, PASP, 94, 244

Hawley, S. L., Gizis, J. E., \& Reid, I. N. 1996, 112, 2799

Henry, T. J., Ianna, P. A., Kirkpatrick, J. D., \& Jarei $\beta$, H. 1997, AJ, 114,388

Henry, T. J., Ianna, P. A., Costa, E., Mendez, R., et al. 1999, The Cerro Tololo Parallax Investigation (CTIOPI), http://www. chara.gsu.edu/ thenry/CTIOPI/

Henry, T. J., Walkowicz, L. M., Barto, T. C., et al. 2002, AJ, 123, 2002

Henry, T. J., et al. 2003, in preparation

Kirkpatrick, J. D., McGraw, J. T., Hess, T. R., Liebert, J., \& McCarthy, D. W. 1994, ApJS, 94, 749

Landolt, A. U. 1992, AJ, 104, 340

Leggett, S. K. 1992, ApJS, 82, 351

Luyten, W. J. 1957, A Catalogue of 9867 Stars in the Southern Hemisphere with Proper Motions Exceeding 0.2 arcsec Annually (Minneapolis, Minnesota: Lund Press)

Méndez, R. A., \& Ruiz, M. T. 2001, ApJ, 547, 252

Patterson, R. J., Ianna, P. A., \& Begam, M. C. 1998, AJ, 115, 1648

Schmidt-Kaler, Th. 1982, in Landolt-Bornstein, New Series, vol. 2, Subvolume b (Springer-Verlag, Berlin), 18

Schneider, H. 1996, in Landolt-Bornstein, New Series, Extension to vol. 2, Subvolume B (Berlin: Springer-Verlag), 20

Sion, E. M., Fritz, M. L., McMullin, J. P., et al. 1988, AJ, 96, 251

Wroblewski, H., \& Costa, E. 2001, A\&A, 367, 725

Wroblewski, H., \& Torres, C. 1991, A\&AS, 91, 129

Wroblewski, H., \& Torres, C. 1997, A\&AS, 122, 447 